\title{
Proceeding
}

Supplementary Issue: Summer Conferences of Sports Science. Costa Blanca Sports Science Events, 25-26 September 2020. Alicante, Spain.

\section{Exploring factors that influence participation in intradialytic physical exercise for haemodialysis users: A phenomenological qualitative study}

\author{
PAULA ISABEL MOSCOSO 4 , CAMILA FERNANDA MADRID, MARIA LUISA GAJARDO \\ Physiotherapy Department, Austral University of Chile, Valdivia, Chile
}

\begin{abstract}
Background: Most of haemodialyzed users are sedentary, because of these the practice of intradialytic physical exercise is fundamental. Programs of physical exercise have been implemented on several dialysis units, but there is still low participation, so it is necessary to find the factors that are influencing participant decisions. Objective: Exploring factors that influence haemodialysis users participation in intradialytic physical exercise. Materials and methods: A phenomenological qualitative study was carried out applying semi-structured interviews to 24 people among patients, family members and health care team. The Colaizzi method was used for data analysis. Results: Three main themes appeared, facilitators, positive impacts, and barriers. Among the facilitators, a favourable social environment, an assertive physical therapy treatment, effective communication and self-care awareness were identified. On the positive impacts, physical impact and quality of life were perceived and, finally, participation barriers included physical and psychosocial factors, and haemodialysis treatment barriers. Conclusions: The present study shows that both facilitators and positive impacts perceived by the users, encourage participation in intradialytic physical exercise, and the barriers decrease it. It seems that to raise participation in intradialytic physical exercise programs, it is necessary to find the local factors influencing participation, enhance facilitators and decrease the existing barriers.

Keywords: Exercise; Renal dialysis; Perception; Patient participation.

Cite this article as:

Moscoso, P.I., Madrid, C.F., \& Gajardo, M.L. (2020). Exploring factors that influence participation in intradialytic physical exercise for haemodialysis users: A phenomenological qualitative study. Journal of Human Sport and Exercise, 15(4proc), S1427-S1436. doi:https://doi.org/10.14198//hse.2020.15.Proc4.39

Corresponding author. Physiotherapy Department, Universidad Austral de Chile, Rudloff 1650, Valdivia, Chile. https://orcid.org/0000-0003-2893-1213

E-mail: p.moscoso@uach.cl

Abstract submitted to: Spring Conferences of Sports Science. Costa Blanca Sports Science Events, 19-20 June 2020. Alicante, Spain.

JOURNAL OF HUMAN SPORT \& EXERCISE ISSN 1988-5202

(C) Faculty of Education. University of Alicante

doi:10.14198/jhse.2020.15.Proc4.39
\end{abstract}




\section{INTRODUCTION}

In the last decades in Chile, the number of people suffering from chronic kidney disease (CKD) has increased exponentially, representing a high cost for their families and the country.

It is possible to find that more than $70 \%$ of haemodialysis users present joint pain, fatigue, myoclonus, weakness and muscle spasms, in addition to a sedentary lifestyle that limit their functional capacity and constitute one of the main causes of low physical capacity.

The National Kidney Foundation recommends physical exercise for dialysis users with a duration of 30 minutes at moderate intensity at least 3 times a week, since it is safe and can improve symptoms secondary to haemodialysis $(\mathrm{HD})$ treatment. This should be prescribed by physiotherapists, according to a previous evaluation with tools such as the 6-minute walk test, the standing and sitting test, and gait speed, among others. It should be noted that physiotherapists are the main source of support during therapy, the exercise program usually lasts between 3 to 12 months with a duration that ranges between 20 and 110 minutes, which includes aerobic, resistance or mixed work, that can range from low to high intensity according to the health condition of each patient (Dorcas, et al 2018; Johansen \& Painter, 2012; Heiwe \& Jacobson, 2011).

Some of the effects of physical exercise at a physiological level are the improvement in parameters such as VO2 max., heart rate variability, increase in muscle strength, it also favours the release of endorphins and thus muscle and mental relaxation, being a protective factor against the depression that these patients usually suffer. (Johansen \& Painter, 2012; Smart \& Steele, 2011; Zhu et al, 2017).

Regarding the social field, intradialysis physical exercise allows interaction with other users, strengthening interpersonal relationships, improving autonomy, sense of identity and responsibility for self-care (Thompson et al, 2016).

Despite the evidence of the effectiveness of intradialysis physical exercise, not all dialysis units have physiotherapists to perform this intervention. Furthermore, in the units that currently have this resource, there is a low user participation in intradialysis physical exercise programs.

Therefore, the objective of this study is to explore in depth the factors that influence decision-making regarding user participation in intradialysis exercise programs, in order to be able to intervene in them and improve participation levels.

\section{MATHERIAL AND METHODS}

A phenomenological qualitative study was carried out with a study population made up of haemodialysis patients, relatives and the dialysis unit health team of Valdivia's Hospital during the second half of 2019. A sample of 24 people was obtained where there were no criteria restriction and was determined by theoretical saturation. The study sample consisted of 11 patients ( 5 of them exercised and 6 did not), 5 family members and 8 health care workers (1 nephrologist, 6 nurses and 1 nurse technician).

Before interviewing the study sample, they were informed about the ethical approval of the project and they were asked to sign an informed consent.

Semi-structured interviews were carried out and they were compiled in audio through the Mlab model 7543. 
Each interview lasted approximately 25 minutes and were performed in different haemodialysis shifts. Patients, health care team and relatives were interviewed in different areas of the Dialysis Unit. Subsequently, the interviews were transcribed and stored in the Microsoft Word program, directly and reliably by each one of the researchers, respecting the confirmability criterion.

To complement the analysis of the phenomenon, a direct non-participant observation was carried out, using a notebook for recording and finally it was organized and included in a field diary.

For the analysis, the Colaizzi method was used, which considered the following:

At the beginning, the analysis was carried out independently by each researcher and the information was compared to reach a consensus of the findings reported in the study in order to increase the validity respecting the triangulation criterion, then the relevant statements were extracted originating formulated meanings which were organized into categories forming three emerging themes, in order to make an exhaustive description. After this, the participants were contacted and the description of 20 of them were validated in person, where no additional information was added.

\section{RESULTS}

24 interviews were conducted with patients, relatives and the health care team. The mean age of the participants was 52 years (26-76 years), $70.8 \%$ of the sample corresponded to women $(n=17)$ and $29.2 \%$ to men $(n=7)$ with a level of schooling in patients from incomplete basic to higher education, in relatives from incomplete basic to high school and the health care team higher education. It should be noted that the patients who exercised had an average of exposure of 18 months with a minimum time of 2 weeks and a maximum of 5 years.
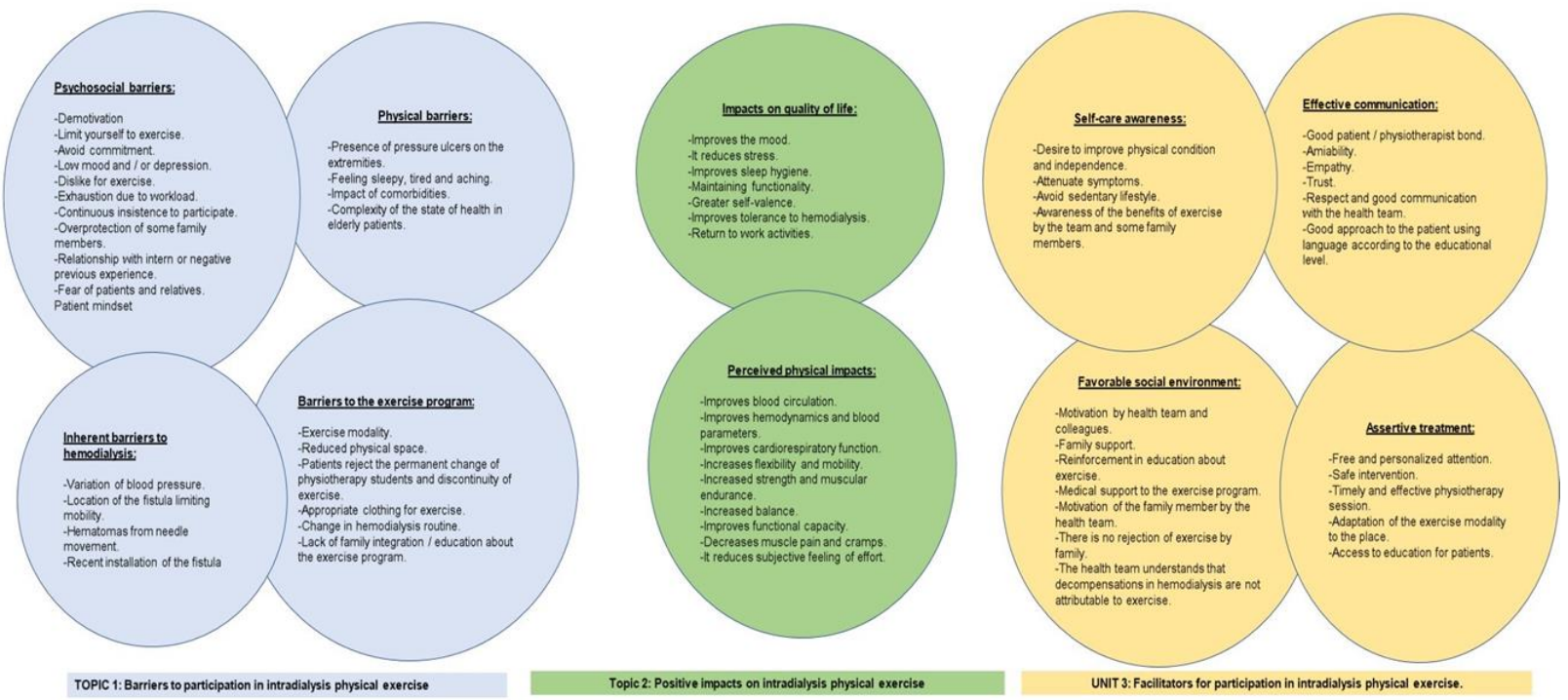

Figure 1. Thematic map of Perception on user participation in intradialysis physical exercise. 
When analysing the interviews, 261 significant statements were extracted, which were grouped into 3 main topics and 10 categories that reflect the perception of user participation in intradialysis physical exercise and the factors influencing participation. (Figure 1).

The first topic was barriers to participation in intradialysis physical exercise, which included the categories "physical barriers", "psychosocial barriers", "barriers to kinetic intervention" and "barriers inherent to haemodialysis". The second topic was positive impacts on intradialysis physical exercise, which incorporated the categories "perceived physical impacts" and "impacts on quality of life". The third topic was facilitators for participation in intradialysis physical exercise that included the categories "favourable social environment", "assertive kinesic treatment", "effective communication" and "self-care awareness". Meaningful quotes were chosen to demonstrate the main categories and themes.

\section{First topic: Barriers to participation in intradialysis physical exercise}

Physical barriers

The main reasons why patients stop exercising, are the presence of cardiac alterations, fatigue, complexity of the state of health, frequent hospitalizations, presence of ulcers in the extremities and body pain, the latter two being also reported by relatives. While those who refuse to participate suffer from intermittent claudication, alterations in the upper extremities and in some cases they must work, which alters the quality of sleep, decreasing their energy, and finally they prefer to sleep during haemodialysis. The aforementioned coincides with the perception of the health care team, which also points out in some cases the association of muscle cramps with physical exercise and they report that, currently in the dialysis unit, there are a large number of older adults with a complex state of health which leads them to reject exercise.

\section{Psychosocial barriers}

Patients who do not exercise believe that having more than one pathology can be risky and they and their family members feel fear, despite having adequate implements and education about the benefits of exercise, in addition to having low mood, demotivation and even depression. Some patients report not having done any sports or physical activity, since they do not like it and that is why they do not want to commit to exercising or to overstress themselves physically, in addition to feeling unable to participate, especially if they have to do other tasks during the day, a decision that is respected by their family members since they understand their situation. Finally, some of them do not like to modify the dialysis routine since the exercise schedule coincides with the delivery of a snack.

At the same time, the health care team believes that there is a difficulty in changing the mentality of patients, especially in those who have not performed physical exercise before or have had previous negative experiences with a physiotherapist.

Finally, the perception of the family members is that on some occasions there is a lot of insistence on the part of physiotherapy students for the patients to exercise, they also indicate that they are unaware of the benefits of intradialysis physical exercise, which leads them to act in an overprotective manner.

Barriers to kinesic intervention

The reduced physical space, the modality and the clothing used for the exercise practice, are not well accepted by some patients.

In general, there is rejection of the permanent changes of physiotherapy students, since bonds of trust are created between them, and the suspension of the intradialytic exercise program during the summer season, 
this is also perceived by the health care team who reports that there is an emotional breakdown and that the evaluation process is repetitive, which generates a bad response from the patients. The health care team also considers that there is a lack of integration and education for family members about the intradialysis physical exercise program, which is also perceived by the latter.

\section{Barriers inherent to haemodialysis}

As perceived by all patients, there is concern due to the presence of the fistula, which limits the movement of the extremities, the location of the needle that can cause bruising when moving, and the variation in blood pressure, especially in the case of hypotension during exercise

At the same time, the health team recommends not exercising only in those patients with recently installed fistulas to avoid bruising.

\section{Second topic: Positive impacts on intradialysis physical exercise. Perceived physical impacts}

When performing intradialysis physical exercise, patients and family members perceive a decrease in body pain and muscle cramps, greater flexibility, improvement in limb circulation and cardiorespiratory function, as well as increased mobility that makes them feel more active and agile. Likewise, the health team mentions that there is better tolerance to dialysis, from the hemodynamic point of view, observing favourable changes in the parameters of blood tests, in addition to an increase in strength, muscular endurance, balance and lung capacity, which is This is reflected in an improvement in functional capacity, reducing the subjective feeling of exertion, especially in cases of patients more compromised in their state of health, which has even led them to go from being in a wheelchair to walking independently.

\section{Impacts on quality of life}

Patients report that exercising improves sleep hygiene and decreases headache, allowing them to resume daily activities without the need for technical aids, increasing their self-valence, this is also recognized by those patients who do not exercise and their families who also notice positive changes in mood and see exercise as an alternative to reduce sedentary lifestyle in patients in whom it has increased due to the long time they remain on haemodialysis. This is reaffirmed by the health team who also observe that patients are not so dependent on their families and some even resume their work generating greater adherence to exercise because they are significant changes for them.

\section{Third topic: facilitators for participation in intradialysis physical exercise}

Favourable social environment

Patients perceive family support for the performance of intradialysis physical exercise, since they see their progress and believe that a change in sedentary behaviour is necessary, coming to understand that postexercise discomfort is the product of physical deconditioning, along with it, they feel motivated by physiotherapists, nurses and even dialysis roommates.

On the other hand, those who chose not to participate in the exercise program, say that their family respects this decision and that if they change their mind they would be supported, which is confirmed by the interviewed relatives.

From the perspective of the health care team, they see that there is a better disposition of younger patients, which encourages others to participate. In addition, they know the benefits of intradialysis exercise, so they support its performance, understanding that decompensation and discomfort are not attributable to this intervention and educate patients about this in order to convince them. 
Assertive physiotherapy treatment

Before making the decision to exercise, patients mention that they are given education about the benefits of it, in addition to the fact that this intervention is free, timely, personalized, effective and safe. Patients feel that this intervention optimizes time on dialysis, which coincides with the opinion of family members and the health care team, the latter also point out that the exercise modality is adjusted to the characteristics of the place, they provide more comfortable chairs for the patients who perform the exercise and in case they need to be evaluated, the schedules are coordinated with the physiotherapist.

\section{Effective communication}

The patients indicate that the kind treatment of the physiotherapist and physiotherapy students is very important since a pleasant interaction is generated during the session, which changes the opinion about this activity.

It should be noted that nurses and doctors perceive that the physiotherapists are empathetic, respectful and maintain good communication within the unit, using a language according to patients educational level, especially at the initial approach, all of this is important to establish a relationship of trust.

\section{Self-care awareness}

When patients have been exercising for a long time, they show a decrease in their symptoms and an improvement in their physical condition, this motivates them since they feel more independent. The health care team mentions that patients are aware of how beneficial it is to exercise avoiding a sedentary lifestyle, an opinion that is also shared by family members. (Figure 2 ).

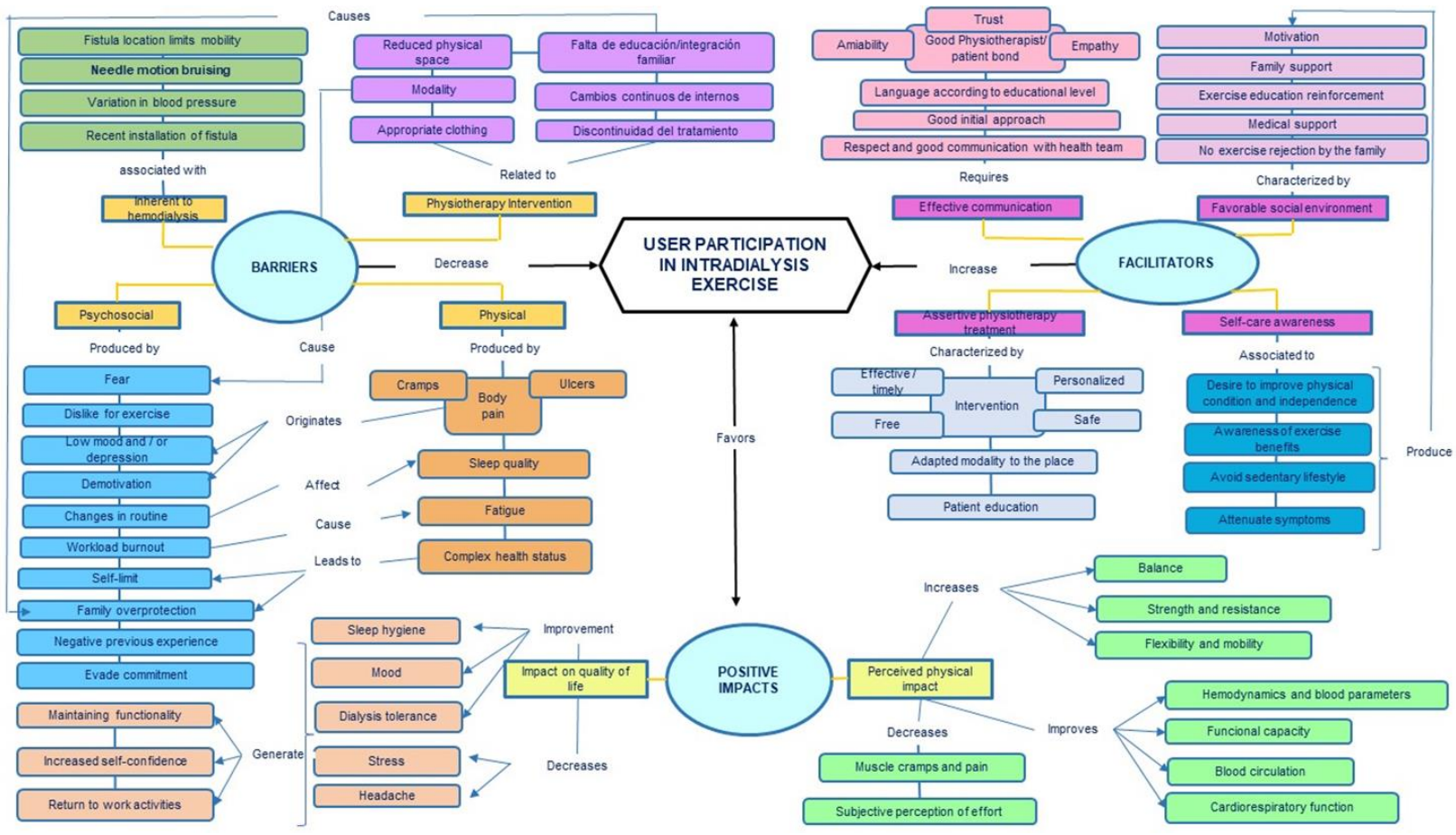

Figure 2. Semantic network on the three emerging themes. 


\section{DISCUSSION}

Although there is sufficient evidence to support the benefits and positive effects of intradialysis physical exercise, at the national level there is little implementation of these in the different units. This is related to multiple factors mainly associated with the fact that this area in Chile has not yet been very developed and there is no support from the health system for this. On the other hand, user participation in existing programs depends on different factors associated with the patient's social and family environment that can directly and indirectly influence decision-making for exercising.

The present study was carried out with the objective of exploring the factors that influence user participation in intradialysis physical exercise programs, from which three common themes emerged for the groups of patients, families and the health care team.

The results of the present study are comparable with those of other authors, finding similarities in the facilitators for participation in intradialysis physical exercise. Thompson et al. (2016) showed that the staff and the physiotherapist are identified as the main sources of support, since they motivate patients to participate by giving them words of encouragement. In addition, most of the patients highlighted the support of the physiotherapist relying on his experience, knowledge and technical instruction interpreted as an emotional meaning. Manisha et al. (2016) found that patients felt self-motivated to exercise as a result of experiencing positive benefits when achieving goals to improve their health or recognizing the physical deterioration resulting from haemodialysis.

A strong relationship of trust between the patients, their doctors and dialysis staff was also identified as a relevant motivating factor, along with encouragement from family, friends and other dialysis patients. The dialysis staff was key to the successful implementation of the physical exercise program, since they had an attitude and willingness to support said program, considering it safe and feasible, adopting the role of stimulating, motivating and monitoring the patient during the session, providing assistance This is why it was easily adapted, without perceiving an increase in the workload, which was also pointed out by Regolisti et al (2018) who found that the support provided by the dialysis staff could increase the perception of well-being of the patients already in turn promoting an active lifestyle that persists over time.

Incorporating an activity into haemodialysis generated distraction and the participants felt the time shorter, which led to an optimization of dialysis. In addition, since the exercise is individualized, the importance they give to it increases. These findings are comparable to those obtained by Delgado et al. (2012), who highlight that the majority of the participants agreed that, by increasing physical exercise, the sedentary lifestyle decreases with less risk to health and that they would be interested in doing it since the time was shortened during dialysis, which is essential for good adherence to the exercise program. On the other hand, Kendrick et al. (2019) highlighted the interest of patients in receiving information about the benefits of physical exercise in order to have greater awareness of the meaning of self-care, which influences their decision to exercise or not.

Finally, Heiwe et al. (2012) showed patients satisfaction when they saw an improvement in their physical well-being since the stiffness was reduced as a result of the long period of immobilization during the haemodialysis treatment, which functioned as a facilitating experience for the continuity of the exercise. Likewise, Moorman et al. (2019) pointed out that the main benefits of an intradialysis exercise program were the improvement of energy and strength, in addition to the improvement in sleep, along with the maintenance of independence and longevity. 
Regarding the impact generated by the participation and performance of intradialysis physical exercise, the results can be compared with that shown by Young et al. (2015) where the patients perceived that their functional abilities improved such as the ability to walk, physiological and psychological health (control of blood pressure and better mood) and reduction of symptoms. This was evidenced by reassessment where exercise performance increased and improvements were observed in their daily life. Staff were highly motivated by the benefits they saw for themselves or those reported by patients, dispelling misconceptions about patient capabilities and increasing staff involvement. Heiwe et al (2012) reported that the patients did not relate cramps suffered to intradialysis physical exercise and described it as a common symptom during the dialysis session. In fact, some patients experienced less leg cramps and restless leg sensations after exercising. Manisha et al. (2016) showed that the participants perceived an improvement in their health and well-being, being beneficial for cardiovascular health, energy level, muscle strength, balance and some of them commented that there were benefits for mental health such as the reduction of the depression, stress and greater sense of achievement, which is similar to what was perceived in our study by the majority of its participants.

Manisha et al. (2016) also refer to the barriers to participation in intradialysis physical exercise related to logistical or patient-specific factors such as fatigue, presence of other comorbidities, fear of falling, lack of options in the modality of intradialysis physical exercise, together with barriers associated with haemodialysis such as fear of injury to the fistula due to movement, needle displacement and infiltration, in addition to instability in blood pressure, limitations due to the inability to use the access arm, cramps during exercise and the change in the haemodialysis routine which is perceived by patients. Clarke et al. (2015) also found that comorbidities such as hypertension, respiratory disease; Mellitus diabetes; Musculoskeletal and mental health diseases, in addition to symptoms such as dyspnoea, joint pain, low back pain and fatigue prevailed as barriers during their study. Delgado et al. (2016) also reported barriers which were fatigue on dialysis and interdialysis days, the presence of ulcers in the extremities and demotivation, indicating some patients feel "too old" to participate in physical exercise. The above was also identified by Fiaccadori et al. (2014), in addition to the feeling of sadness and previous physical condition, since those physically inactive subjects showed a higher prevalence of barriers associated with participation in intradialysis exercise.

Some results could not be compared or contrasted with other studies because they are unique findings of the dialysis unit of the Hospital Base Valdivia and are related to the discontinuity of the intradialysis exercise program during the summer season, because there is no permanent physiotherapist in the unit.

In this research, family members were included where the results could not be compared with other studies, since no others were found where the perception of these was considered, therefore our research will be useful for future studies since it is the first to describe the influence that family members could have on users participation and will also allow to improve the exercise programs, taking into account the beliefs and assumptions about this kind of intervention, providing the security and trust necessary for participation.

The obtained information cannot be extrapolated because of the type of study, since qualitative phenomenological research seeks to describe and understand the behaviour of people from the lived experience, that is, from within the phenomenon to be studied, and this may vary depending the context where the investigation is developed. 


\section{CONCLUSIONS}

The present study shows that both facilitators and positive impacts perceived by the users, encourage participation in intradialytic physical exercise, and the barriers decrease it. It seems that to raise participation in intradialytic physical exercise programs, it is necessary to find the local factors influencing participation, enhance facilitators and decrease the existing barriers.

The limitations presented in this study were the difficulty in recruiting family members due to the fact that many of the patients attend haemodialysis alone, traveling in institutional vehicles or ambulances from other locations and several accompanying family members did not show interest in participating. In addition, in the last step of validating the information, four participants were not willing to meet again with the researchers.

In the first interviews, an inadequate drafting of some questions was evidenced, which were modified and were not considered in the results. Along with this, the reduced number of family members prevented the saturation of the information, these being the main biases of the study.

More studies are needed to identify other factors that could influence user participation in intradialysis exercise.

\section{REFERENCES}

Clarke, A. L., Young, H. M. L., Hull, K. L., Hudson, N., Burton, J. O., \& Smith, A. C. (2015). Motivations and barriers to exercise in chronic kidney disease: A qualitative study. Nephrology Dialysis Transplantation, 30(11), 1885-1892. https://doi.org/10.1093/ndt/gfv208

Delgado, C., \& Johansen, K. L. (2012). Barriers to exercise participation among dialysis patients. Nephrology Dialysis Transplantation, 27(3), 1152-1157. https://doi.org/10.1093/ndt/gfr404

Dorcas, T. M., Youth, T. B., Atuhaire, C., Priebe, G., \& Cumber, S. N. (2018). Physiological and psychosocial stressors among hemodialysis patients in the Buea Regional Hospital, Cameroon. Pan African Medical Journal, 30, 30-49. https://doi.org/10.11604/pami.2018.30.49.15180

Fiaccadori, E., Sabatino, A., Schito, F., Angella, F., Malagoli, M., Tucci, M., Cupisti, A., Capitanini, A., \& Regolisti, G. (2014). Barriers to physical activity in chronic hemodialysis patients: A single-center pilot study in an Italian dialysis facility. Kidney \& Blood Pressure Research, 39(2-3), 169-175. https://doi.org/10.1159/000355793

Heiwe, S., \& Jacobson, S. H. (2011). Exercise training for adults with chronic kidney disease. Cochrane Database of Systematic Reviews, 10. https://doi.org/10.1002/14651858.cd003236.pub2

Heiwe, S., \& Tollin, H. (2012). Patients' perspectives on the implementation of intra-dialytic cycling a phenomenographic study. Implementation Science, 7(1), 7-68. https://doi.org/10.1186/1748-5908-7$\underline{68}$

Jhamb, M., McNulty, M. L., Ingalsbe, G., Childers, J. W., Schell, J., Conroy, M. B., Forman, D. E., Hergenroeder, A., \& Dew, M. A. (2016). Knowledge, barriers and facilitators of exercise in dialysis patients: a qualitative study of patients, staff and nephrologists. BMC Nephrology, 17(1), 17. https://doi.org/10.1186/s12882-016-0399-z

Johansen, K. L., \& Painter, P. (2012). Exercise in individuals with CKD. American journal of kidney diseases: the official journal of the National Kidney Foundation, 59(1), 126-134. https://doi.org/10.1053/i.ajkd.2011.10.008 
Kendrick, J., Ritchie, M., \& Andrews, E. (2019). Exercise in Individuals With CKD: A Focus Group Study Exploring Patient Attitudes, Motivations, and Barriers to Exercise. Kidney Medicine, 1(3), 131-138. https://doi.org/10.1016/j.xkme.2019.03.004

Moorman, D., Suri R., Hiremath S., Jegatheswaran J., Kumar T., Bugeja A., \& Zimmerman T. (2019). Benefits and Barriers to and Desired Outcomes with Exercise in Patients with ESKD | American Society of Nephrology. (s. f.).14 (2) 268-276; https://doi.org/10.2215/CJN.09700818

Regolisti, G., Maggiore, U., Sabatino, A., Gandolfini, I., Pioli, S., Torino, C., Aucella, F., Cupisti, A., Pistolesi, V., Capitanini, A., Caloro, G., Gregorini, M., Battaglia, Y., Mandreoli, M., Dani, L., Mosconi, G., Bellizzi, V., Di lorio, B. R., Conti, P., \& Fiaccadori, E. (2018). Interaction of healthcare staff's attitude with barriers to physical activity in hemodialysis patients: A quantitative assessment. PLOS ONE, 13(4), e0196313. https://doi.org/10.1371/journal.pone.0196313

Smart, N., \& Steele, M. (2011b). Exercise Training in Hemodialysis Patients: A Systematic Review and Meta-Analysis. Nephrology, 16(7), 626-632. https://doi.org/10.1111/j.1440-1797.2011.01471.x

Thompson, S., Tonelli, M., Klarenbach, S., \& Molzahn, A. (2016). A Qualitative Study to Explore Patient and Staff Perceptions of Intradialytic Exercise. Clinical Journal of the American Society of Nephrology, 11(6), 1024-1033. https://doi.org/10.2215/cjn.11981115

Young, H. M. L., Hudson, N., Clarke, A. L., Dungey, M., Feehally, J., Burton, J. O., \& Smith, A. C. (2015). Patient and Staff Perceptions of Intradialytic Exercise before and after Implementation: A Qualitative Study. PLOS ONE, 10(6), e0128995. https://doi.org/10.1371/journal.pone.0128995

Zhu, F.-X., Zhang, X.-Y., Ding, X.-K., \& Han, B. (2017). Protective effect of regular physical activity on major depressive episodes in patients with early stages of chronic kidney disease. Renal Failure, 39(1), 602-606. https://doi.org/10.1080/0886022x.2017.1361833

This work is licensed under a Attribution-NonCommercial-NoDerivatives 4.0 International (CC BY-NC-ND 4.0). 